Lingüística

Vol. 33-2, diciembre 2017: 55-68

ISSN 2079-312X en línea

DOI: $10.5935 / 2079-312 X .20170017$

\title{
TÉRMINOS DE PARTES DEL CUERPO HUMANO EN MEHINAKU (ARAWAK)
}

\author{
TERMS OF HUMAN BODY PARTS IN MEHINAKU (ARAWAK)
}

Ángel Corbera Mori

Universidad Estadual de Campinas (UNICAMP)

angel@unicamp.br

\section{Resumen}

El cuerpo humano es un sistema jerárquico constituido de partonomía corporal, definido por rasgos básicos y universales, caracterizados por un conjunto jerárquico de relaciones, del tipo "X es parte de". Por ejemplo, las uñas son partes de los dedos, los dedos son partes de la mano (Andersen 1978). Ya en las clasificaciones taxonómicas, los elementos de una categoría son conceptuados como "X es un tipo de", por ejemplo, la rosa es un tipo de flor, la trucha es un tipo de pez de río (McClure 1975). Considerando estos dos tipos de clasificación, se presenta una breve descripción de la partonomía en la lengua mehinaku (arawak). Se analizan los términos de las partes del cuerpo considerando la estructura morfológica de esta lengua y su ocurrencia en construcciones de posesión nominal. Los datos lingüísticos presentados en este texto son el resultado de trabajos de campo realizados en aldeas del pueblo mehinaku.

Palabras clave: Lenguas amerindias, Familia lingüística arawak, Lengua mehinaku. Lenguas del Alto Xingú.

The human body is a hierarchical system constituted of corporal partonomy, defined by basic and universal traits of the human conception, characterized by a hierarchical set of relations, of type " $X$ is part of". For example, the nails are parts of the fingers, the fingers are parts of the hand (Andersen 1978). Already in taxonomic classifications, the elements of a category are conceptualized as "X is a type of", for example, the rose is a type of flower, trout is a type of river fish (McClure 1975). Considering these two types of classification, a brief description of the partonomy in Mehinaku language (Arawak) is presented. The terms of parts of the body are approached considering the morphological structure of this language and its occurrence in constructions of nominal possession. The linguistic data presented in this text are the result of fieldworks conducted in villages of the Mehinaku people. 
Key words: Amerindian languages, Arawak language family, Mehinaku language. Languages of the Upper Xingú.

Recibido: 28/01/2017

Aceptado: 01/07/2017

\section{Introducción}

Este artículo trata someramente de la organización léxica de las partes externas e internas del cuerpo humano en la lengua y cultura del pueblo mehinaku. En el análisis e interpretación de los datos, se considera la estructura morfosintáctica de la formación de palabras en esta lengua. La organización léxica en la lengua mehinaku corresponde a las unidades morfosintácticas que los hablantes de cualquier lengua atribuyen a las palabras y sus correspondientes referentes definidos formal y conceptualmente como "partes del cuerpo humano". Los términos que identifican a las partes del cuerpo humano en la lengua y cultura de la sociedad mehinaku no siempre coinciden con las partes del cuerpo humano vehiculados en lenguas europeas, como español o portugués.

Por el conjunto de datos léxicos registrados y analizados hasta el presente, se percibe que los lexemas correspondientes a los términos de partes del cuerpo pueden ser identificados siguiendo criterios tanto formales cuanto nocionales, que justamente, se correlacionan con la identificación de las categorías léxicas de la lengua mehinaku. En un trabajo anterior (Corbera Mori 2011), se mostró que los diferentes tipos de ítems de la categoría léxica nominal pueden ser subcategorizados en nombres alienables y nombres inalienables, diferencias que son identificadas en estructuras de posesión nominal. Someramente, los nombres considerados inalienables son subcategorizados obligatoriamente como [+POSESIÓN] y los nombres alienables como [-POSESIÓN]. Una diferencia formal entre estas dos subcategorías se da morfológicamente. Los nombres considerados inalienables, para ocurrir en una relación de posesión, precisan solo de la mediación de los clíticos pronominales de persona/número poseedora; en contraste, los nombres de la subcategoría alienables, además de los clíticos pronominales de persona/número poseedora, deben ser marcados obligatoriamente con los diferentes sufijos que indican 'posesión'. Los términos nominales que se relacionan con las partes del cuerpo humano se encuentran en la subcategoría de los nombres subcategorizados por el rasgo [+POSESIÓN].

\section{El pueblo y la lengua mehinaku}

El mehinaku es una lengua amazónica hablada por un pueblo originario conocido por la sociedad nacional por el mismo nombre. La población mehinaku es de, aproximadamente, 286 personas (Instituto Socioambiental (ISA) 2017) y se autoidentifican como Imiehünaku, distribuyéndose 
actualmente por 4 aldeas: la primera, Uyaipiyuku [ujaipjuku], es la más antigua, y otras tres que se formaron en los últimos diez años: Utawana, aldea que se localiza al lado del Puesto Indígena de Vigilancia (PIV), Aturua y Kaupüna. Las cuatro aldeas se ubican a las márgenes del río Kurisevo, al sur del Parque Indígena del Xingú, en la región del Alto Xingú, Estado de Mato Grosso, Brasil. La lengua Imiehünaku iayaka 'nuestra lengua mehinaku' hablada por este pueblo integra junto con las lenguas waurá [wa'uza] y yawalapiti [jawala'pihi] la familia lingüística arawak, subgrupo xinguano (Aikhenvald 1994) o arawak central, de acuerdo a la clasificación de Payne (1991)

\section{El léxico de las partes del cuerpo}

La colecta de los términos relacionados a las partes del cuerpo humano en la lengua mehinaku se realizó durante diversos períodos de estadía y trabajos de campo en la aldea indígena Utawana. Los datos primarios fueron registrados en sesiones de elicitación y en conversaciones informales con el cacique Yahati Mehinaku, además de sus dos hermanos: Anapuatá y Yutá, respectivamente. Datos adicionales fueron proporcionados por Waxamaní Mehinaku, de 21 años de edad, hijo del cacique mencionado. Las elicitaciones fueron realizadas paralelamente a la recolección de datos sobre aspectos gramaticales de la lengua; el registro específico de los términos de partes del cuerpo se hizo considerando las ilustraciones del cuerpo humano masculino y femenino presentados en Enfield (2006).

\subsection{Estructura de la posesión nominal en mehinaku}

Como mencionamos en $\S 1$, los nombres subcategorizados como [POSESIÓN] no precisan de un dependiente poseedor para manifestarse lexicalmente. Sin embargo, cuando ocurren en una relación de posesión, estos tipos de nombres deben obligatoriamente aparecer precedidos por los clíticos pronominales indicadores de la persona poseedora y número, seguidos por morfemas sufijos, que también son obligatorios. Estos sufijos establecen la relación de posesión entre el elemento poseído y el poseedor. La posesión en este tipo de nominales puede ser dividida en cuatro subgrupo o subclases. El primer subgrupo tiene la estructura [clítico pronominal-base nominal-morfema de posesión]. ${ }^{1}$ El morfema de posesión es marcado por el sufijo $\{-l a\}$ y sus realizaciones alomórficas $/-l a \approx-l e \approx-r a \approx-s a /$, que son variantes condicionadas fonológicamente por la última vocal de la raíz nominal. De esta manera, /-le/ ocurre cuando la vocal final de la raíz es /e/, /-ra/ cuando la vocal es /i/, /-sa/ cuando es /i// y /-la/ si la vocal es / $u /$ o/a/. Adicionalmente, la penúltima sílaba de la palabra debe ser tónica. Los ejemplos de (1a-d) muestran este caso.

\footnotetext{
${ }^{1}$ Estos clíticos pronominales, analizados tradicionalmente como prefijos, son formas reducidas de los pronombres personales libres, que ocurren precediendo las bases nominales en estructuras de posesión y también precediendo bases verbales, por ejemplo: nu-tanu'le-su [1prima-FEM] 'mi prima', nu-katumala-'pai [1-trabajar-EST] 'yo trabajo'.
} 
1.

a. u'ku

Formas no poseídas

ma'tapu

'teme

we'he-pe

'maiki

tu'wapi

i'suhi

'pahi

Glosa
'flecha'
'zumbador'
'tapir'
'ceniza'
'maíz'
'estera'
'anzuelo'
'mono'

Poseídas

n-u'ku-la

nu-mata'pu-la

nu-te'me-le

nu-wehe-'pe-le

nu-'maiki-ra

nu-tuwa'pi-ra

ni-su'hi-sa

nu-pa'hi-sa
Glosa ${ }^{2}$

'mi flecha'

'mi zumbador'

'mi tapir'

'mi ceniza

'mi maíz'

'mi estera'

'mi anzuelo'

'mi mono'

El segundo suugrupo de nombres alienables se caracterizan por recurrir al proceso de morfología no concatenativa. Así, el núcleo oral de la última sílaba de la base nominal no poseída ocurre como vocal nasalizada y tónica en una construcción de posesión, como se constata en los ejemplos de (1b).

$\begin{array}{llll}\text { Formas no poseídas } & \text { Glosa } & \text { Poseídas } & \text { Glosa } \\ \text { 'itsa } & \text { 'canoa' } & \text { n-i'tsã } & \text { 'mi canoa' } \\ \text { pu'taka } & \text { 'aldea' } & \text { nu-puta'kã } & \text { 'mi aldea' } \\ \text { i'kiri } & \text { 'paja' } & \text { n-iki'rĩ } & \text { 'mi paja' } \\ \text { i'hì } & \text { 'sal' } & \text { n-i'hĩũ } & \text { 'mi sal' } \\ \text { 'ulu } & \text { 'concha' } & \text { n-u'lũ } & \text { 'mi concha' }\end{array}$

El tercer subgrupo de este tipo de nombres también recurre a la morfología no concatenativa, puesto que las bases nominales, tanto en la forma no poseída como en la poseída, no sufren ninguna modificación en la cadena segmental, excepto que en la forma poseída la última sílaba de la palabra pasa a ser tónica. Este caso se presenta en los ejemplos de (1c).

$\begin{array}{llll}\text { c. Formas no poseídas } & \text { Glosa } & \text { Poseídas } & \text { Glosa } \\ \text { pa'lata } & \text { 'peine' } & \text { pi-piala'ta } & \text { 'tu peine' } \\ \text { pa'nana } & \text { 'plátano' } & \text { nu-pana'na } & \text { 'mi plátano' } \\ \text { e'tene } & \text { 'remo' } & \text { p-ete'ne } & \text { 'tu remo' } \\ \text { 'nete } & \text { 'chaquira' } & \text { nu-ne'te } & \text { 'mi chaquira' } \\ \text { wasa'ju-ti } & \text { 'frijol' } & \text { nu-wasaju-'ti } & \text { 'mi frijol' } \\ \text { i'tfuwi } & \text { 'caracol' } & \text { p-itfu'wi } & \text { 'tu caracol' }\end{array}$

\footnotetext{
${ }^{2}$ Los datos que se presentan en este texto son transcriptos fonológicamente, con base en los símbolos del International Phonetic Alphabet (IPA).
} 
Por último, hay un conjunto de ítems nominales que solo son marcados por los clíticos pronominales de persona/número en la forma poseída, pero sin presentar ninguna modificación en la base respecto de la forma no poseída. En este sentido, no hay un morfema visible que muestre la relación de posesión entre el poseedor y el núcleo nominal poseído. Los siguientes datos (1d) son ejemplos de este tipo de construcción.

$\begin{array}{llll}\text { d. Formas no poseídas } & \text { Glosa } & \text { Poseídas } & \text { Glosa } \\ & \text { 'cazabe' } & \text { p-u'lepe } & \text { 'tu cazabe' } \\ \text { kitu'la-ja } & \text { 'pelota' } & \text { nu-kitu'la-ja } & \text { 'mi pelota' } \\ \text { 'wãjũ } & \text { 'rodillera' } & \text { nu-'wãjũ } & \text { 'mi rodillera' } \\ \text { pe'teşi } & \text { 'roza' } & \text { nu-pe'teşi } & \text { 'mi roza' }\end{array}$

En la subcategoría que engloba a los nombres con el rasgo [+POSESIÓN] se encuentran las unidades léxicas referentes a las partes del cuerpo humano. Los términos de partes del cuerpo pueden ocurrir únicamente con indicación de un poseedor específico, dado por los clíticos pronominales de persona y número. En determinadas ocasiones que, inicialmente, podrían ser analizadas como formas del vocativo ocurren sin los marcadores del poseedor; pero, en este caso, la base correspondiente debe recibir obligatoriamente el sufijo $\{-i\}$ 'ABSOLUTO', morfema que señala la ocurrencia de un referente sin poseedor específico, o 'forma no poseída'.

En consonancia con Enfield (2006) y Meira (2006), en el inventario de los términos que se relacionan con partes del cuerpo hay que considerar dos tipos de categorías: (i) los lexemas simples, formas que son monomorfémicas, (ii) lexemas complejos, que son formas polimorfémicas motivadas semánticamente. Con base en estas características, los términos de partes del cuerpo en la lengua mehinaku serán abordados según su estructura morfológica, en bases nominales simples y bases nominales complejas.

\subsubsection{Bases nominales simples}

Meira (2006: 263), al analizar la complejidad morfológica de los términos de partes del cuerpo en la lengua tiriyó (karib), interpreta los términos simples como bases primitivas, que no son segmentables en unidades menores, y los términos complejos como bases morfológicamente derivadas y con varios grados de plausibilidad sincrónica de segmentación. En esta categoría incluye también estructuras sintagmáticas, o sea, construcciones en que claramente coocurren dos bases independientes.

Así como acontece en tiriyó, en la lengua mehinaku se puede considerar como bases simples los términos de partes del cuerpo que siendo subcategorizadas por el rasgo [+POSESIÓN] no ocurren con los marcadores pronominales que indican la persona/número poseedora. Pero al no ocurrir en su forma poseída, estos exigen la presencia obligatoria del sufijo $\{-i\}$ 'ABSOLUTO', como se muestra en los ejemplos en (2) 
2. Bases no poseídas $\rightarrow\left[[X]_{N^{-}}{ }^{\prime} A B S^{\prime}\right]$

$\begin{array}{ll}\text { ti'w-i } & \text { 'cabeza' } \\ \text { utita-'i } & \text { 'ojo' } \\ \text { hekira-'i } & \text { 'frente' } \\ \text { ju'hia-i } & \text { 'pestañas' } \\ \text { te'we-i } & \text { 'diente' } \\ \text { hala'pa-i } & \text { 'mandíbula' } \\ \text { tulũ-'ĩ } & \text { 'oreja' } \\ \text { henepu-'i } & \text { 'hombro' } \\ \text { wana-'i } & \text { 'brazo' } \\ \text { wišiku-'i } & \text { 'mano' } \\ \text { tetu-'i } & \text { 'rodilla' } \\ \text { tipulu-'i } & \text { 'calcañar/talón' } \\ \text { ma'p-i } & \text { 'vellos del cuerpo' }\end{array}$

En este grupo de bases simples o no derivadas, se incluyen también aquellos términos corporales que solo son prefijados por los clíticos pronominales marcadores de la persona/número poseedora, como se registra en los siguientes ejemplos.

3. Bases con clíticos pronominales prefijados $[C L P R O N M-[X]]_{N}$

$\begin{array}{ll}\text { nu-'kiri } & \text { 'mi nariz' } \\ \text { nu-he'kira } & \text { 'mi frente' } \\ \text { nu-'tiu } & \text { 'mi cabeza' } \\ \text { nu-wi'siku } & \text { 'mi mano' } \\ \text { nu-'puhu } & \text { 'mi cabello (cabello de mujer)' } \\ \text { pi-tsu'lu } & \text { 'tu oreja' } \\ \text { pi-'pijũ } & \text { 'tu cuello, pescuezo' } \\ \text { pi-'jana } & \text { 'tu brazo' } \\ \text { pi-'tfetu } & \text { 'tu rodilla' } \\ \text { pi-'tsewe } & \text { 'tu diente' } \\ \text { i-je'seti } & \text { 'su nalga' } \\ \text { ji-'kiri } & \text { 'nariz de Uds.' } \\ \text { ji-'tfitju } & \text { 'barriga de Uds.' }\end{array}$

\subsubsection{Bases nominales complejas}


Dentro de las bases complejas o derivadas se registran dos posibilidades: (i) bases nominales precedidas por los clíticos pronominales de persona/número poseedora y sufijadas por un clasificador, (ii) bases nominales precedidas por los clíticos pronominales de persona/número poseedora, seguida por otra base nominal, formando un tipo de nominal compuesto, que, a su vez, pueden o no recibir un clasificador. Estos dos tipos de construcción son presentados a continuación.

4. Construcción con clítico pronominal-base simple-clasificador

nu-ta'na-ka

1-ala-CL.largo

'mis costillas'

nu-nupana-'taku

1-pecho-CL.LOC.área/superficie

'mi pecho'

nu-kati-'tapa

1-pierna-CL. racimo

'mi pantorrilla'

pi-tfana-'ti

2-CL.cóncavo-CL.cilíndrico

'tu boca'

pi-piawa-'taku

2-rostro-CL.LOC.área/superficie 'tu rostro, cara'

pi-juhia-'mepe

2-pestaña-CL.apiñado 'tu ceja, cilio'

pi-'trana-'tapa ${ }^{3}$

2.-CL.cóncavo-CL.racimo 'tu muñeca, pulso'

p-uwisiku-'taku

2-mano-CL.LOC.área/superficie 'tu palma de la mano'

i-pĩjũ-naku

3-cuello-CL.LOC.interno/dentro 'su garganta'

i-numa-ta'ka-pi

3-boca(externo)-CL.techumbre-CL.lineal 'su mandíbula'

5. Construcción con clítico pronominal-base compuesta - (clasificador)

nu-kati -'kiri

1-pierna-naríz/pico 'mi canilla'

ni-k-i'sa-pi

1-ATRIB-sangre-CL.lineal 'mi labio'

nu-kapi-tiu-'tari

1-mano-cabeza-CL.redondo 'mi dedo pulgar'

nu-wana-ti'pulu

1-brazo-talón

'mi codo'

\footnotetext{
${ }^{3}$ En esta construcción parece tratarse de la composición de dos clasificadores que forman el sintagma posesivo correspondiente: -tfana es un alomorfo del morfema -kana, un clasificador que indica 'hueco', 'cuenco' 'cosa cóncava', seguido por el clasificador -tapa 'racimo'.
} 
pi-trapi-'tiwi

2-mano-cabeza

pi-tyati-pĩjũ-'naku

2-pierna-pescuezo-CL.interno

pi-ki-tsiu-he'neku

2-pie-cabeza-hombro

i-ki-tsiu

3-pie-cabeza

i-ki-tsiu-'tari

3-pie-cabeza-CL.redondo

i -k-isa-pi-pie-nu'ma-ti

3-ATRIB-sangre-CL.lineal-CL.masa-boca(externo)-CL.cilíndrico 'su bigote'

\begin{abstract}
'tu dedo de la mano'
\end{abstract}
'tu fosa popliteal'

'tu interdigital del pie'

'su dedo del pie'

'su dedo gordo del pie (hallux)'

\section{Términos referidos a las partes internas}

Los términos relacionados a las partes internas del cuerpo humano reflejan la estructuración formal presentada en las secciones anteriores. Algunos ejemplos de estos casos se presentan a continuación.

6. nu-waja'la-pi

1-venas-CL.lineal

'mi vena'

pi-tsiu-naku-'ti

2-cabeza-CL.LOC. interno-CL.semilla

'tu seso'

pi-tsiu-naku-'ti

2-cabeza-CL.LOC.interno-CL.semilla

'tu cerebro'

p-ĩ'wĩ-tsi

2-respiração-CLsemilla/cilíndrico

'tu corazón'

i-'napi

3-hueso

'su hueso'

i-ti-'napu

3-CL.semilla/cilíndrico-camino 'su vagina (Lit. camino del pene)'

i-puku-'ti

3-punta-CL.semente/cilíndrico

'su cabeza del pene'

i -nu'pana

3-hígado

'su hígado'

i-nula-pi-'pie

3-intestino-CL.lineal-CL.masa

'su intestino'

kiri-jãku-'i

naríz-CL.LOC.interno-ABS

'narinas'

hiffu't-i

ano-ABS

'ano' 


$\begin{array}{ll}\text { tulu-naku-'i } & \\ \text { oreja-CL.LOC.interno-ABS } & \text { 'oído' } \\ \text { ula-ta'pa-i } & \\ \text { comida-CL.racimo-ABS } & \text { 'estómago' } \\ \text { pana-i } & \\ \text { clítoris-ABS } & \text { 'clítoris' } \\ \text { ja'la-i } & \\ \text { himen-ABS } & \text { 'himen vaginal' } \\ \text { hauka-'na-i } & \\ \text { bebe-casa-ABS } & \text { 'placenta' }\end{array}$

\section{Términos que indican sustancias del cuerpo}

Los términos que se refieren a sustancias emanadas del cuerpo también se engloban en la categoría de los nominales inalienables [+POSESIÓN], de ahí que, al no ocurrir en una construcción de posesión, estas bases obligatoriamente recibirán el marcador $\{-i\}$ de 'no posesión' o 'ABSOLUTO'. Algunos ejemplos se incluyen a continuación.

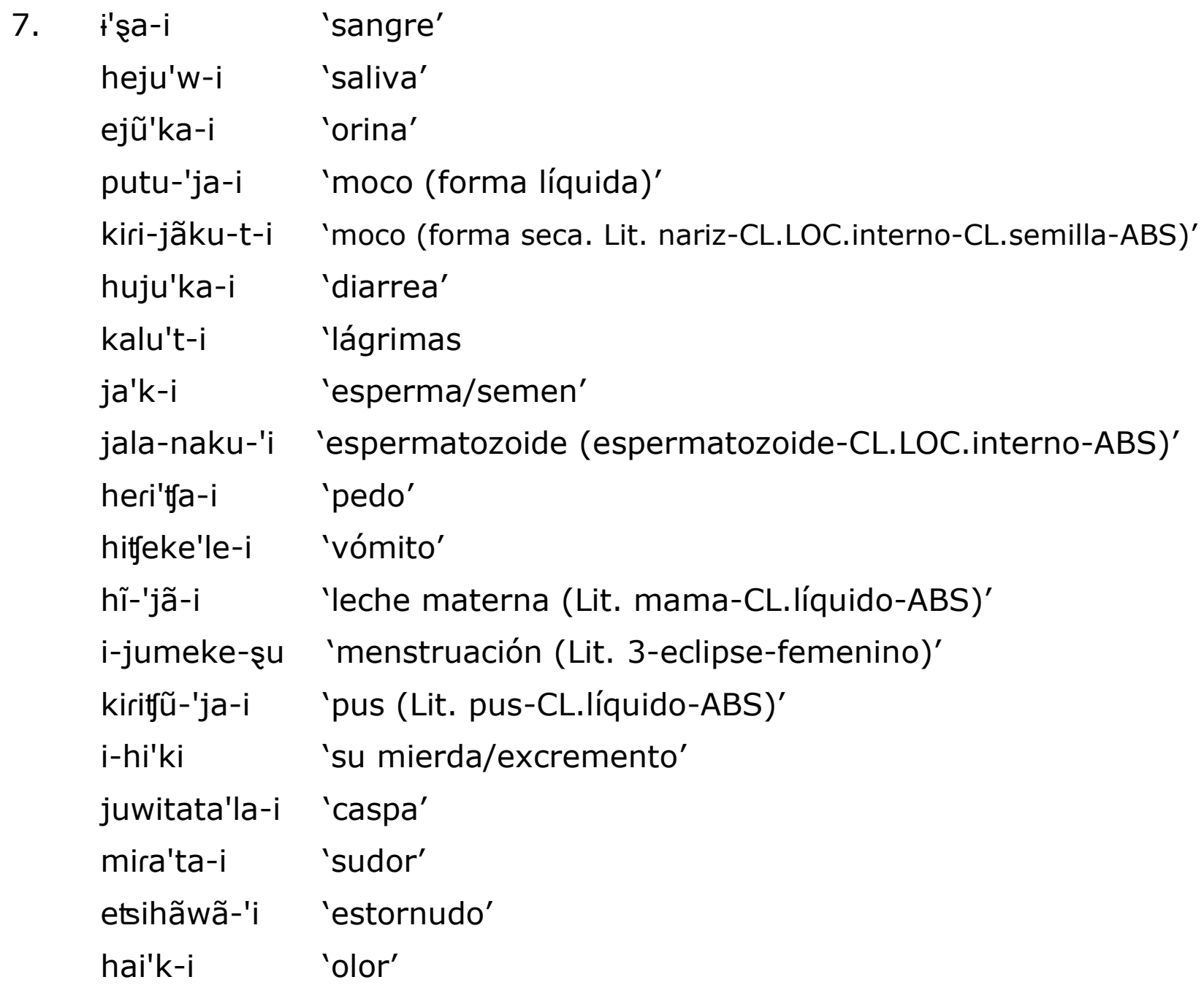




$$
\begin{array}{ll}
\text { maku-'i } & \text { 'gordura' } \\
\text { putu-'ja-i } & \text { 'resfriado (resfriado-CL.líquido-ABS)' } \\
\text { nu-waja'la-pi } & \text { 'mi vena (1-vena-CL.lineal) }
\end{array}
$$

\section{Extensiones de los términos de partes del cuerpo}

Según Enfield (2006), es importante verificar la existencia de términos de partes del cuerpo humano en que sus referencias sean extensibles para nombrar partes del cuerpo de animales. En este caso, resulta relevante hacer una breve comparación de los términos de partes del cuerpo encontrados en la lengua mehinaku con aquellos usados por sus hablantes en la denominación de las partes que ocurren en aves, peces y mamíferos. Al hacerlo, se constató una coincidencia exacta, si bien no con todos los términos, pero con un número alto de los mismos. A continuación, son ejemplificados los términos más recurrentes.

\subsection{En aves}

Algunos de los términos corporales de las aves que son similares en la forma y significado con aquellos del cuerpo humano son los siguientes.

8. i-puti-'tapa

3-pierna-CL. racimo

'su pierna'

i-tunulu-'naku

3-oreja-CL.LOC.interno

'su oído'

i-'kiri

3-nariz

'su pico (Lit. su nariz)'

i-ki-tsapa-'taku

3-pie- racimo-CL.LOC.área

'su planta del pie (del gallo)'

i-kiri-'jãku

3-nariz-CL.LOC.interno

'su narina (del pico)'

i-pijũ-'naku

3-pescuezo-CL.LOC.interno

'su garganta'

i-ta'na-ka

3-ala-CL.largo

'su escápula (Lit. espalda/omóplato)'

in-uti'tai

3-ojo

'su ojo'

i-ki-tsapa-'penu

3-pie-racimo-CL.LOC.sobre

'dorso del pie/pata'

ini-'tfetu

3-rodilla

'su corvejón (Lit. rodilla)' 


\subsection{En mamíferos}

Así como en el caso de las aves, también en los mamíferos, por ejemplo, caballo, buey, vaca, determinados términos corporales se estructuran morfológicamente de la misma forma que sus similares de aquellas partes del cuerpo humano. Esta característica involucra, igualmente, el significado como se puede constatar por las glosas en castellano en los datos registrados a continuación.

9. $\quad \dot{i}$-tu'lũ

3-oreja

'su oreja' (caballo, buey)

i-puti-tapa

3-pierna-racimo

'su pierna' (buey, vaca, caballo)

i-ki-tsapa-'tari

3-pie-CL.racimo-CL.redondo

'su casco, pezuña' (caballo, buey)

i-tana-ka-pi

3-ala-CL.largo-CL.lineal

'su espalda/lomo' (buey, vaca)

'i-ti

3-CL. semilla/cilíndrico

'su pene' (caballo, buey)

'i-hit

3-mama

'su ubre' (Lit. su teta de la vaca)

i-numa-ti-'kapi

3-boca-CL.semilla/cilíndrico-mano 'papada' (buey, vaca)

i-nu'ma-ti

3-boca-CL.semilla/cilíndrico

'morro' (buey, vaca)

i-ha'paja

3-vientre

'su barriga/vientre' (caballo, buey, vaca)

\subsection{En peces}

Como en el caso de las aves y mamíferos, también es posible encontrar paralelismos en la denominación de determinadas partes del cuerpo de los peces. Algunos de estos términos se listan a continuación.

10 i-kiri-'jãku

3-nariz-CL.LOC. interno 'su narina'

i -hekira-'penu

3-frente-CL.LOC.sobre 'su parte superior de la frente del pez'

i-ka'na-ti

3-CL. cóncavo-CL.cilíndrico 'su boca'

in-uti'tai

3-ojo

'su ojo'

i-ta'na-ka-pi

3-ala-CL.largo-CL.lineal 'su dorso/espalda' 


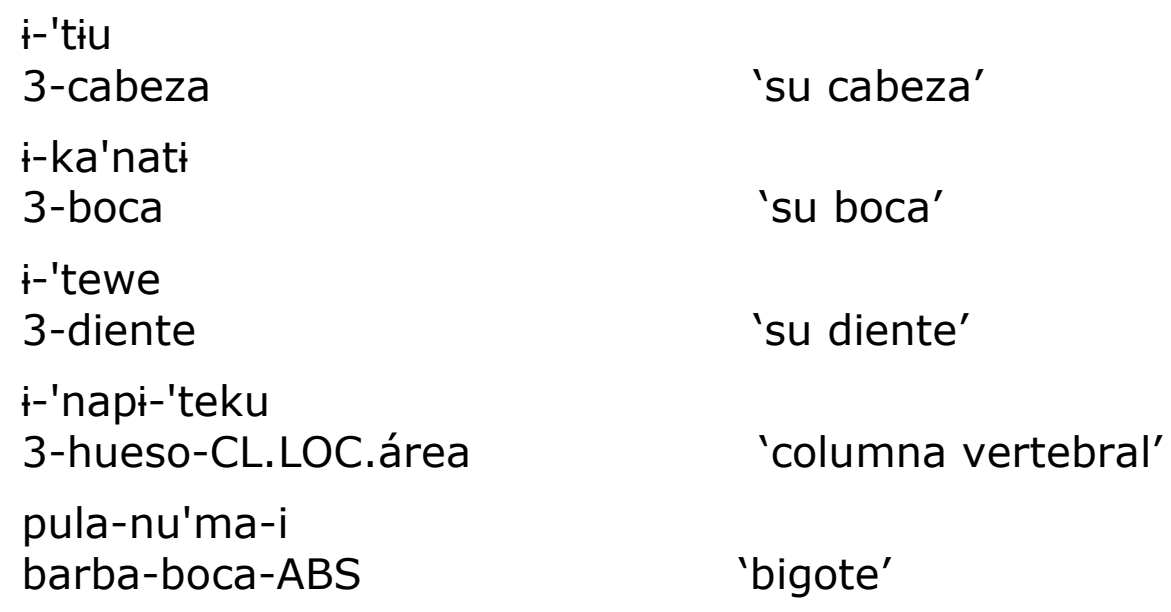

Finalmente, los términos de partes del cuerpo humano tienen su paralelo en las denominaciones de algunas partes de las casas tradicionales construidas a base de madera, paja y lianas. También algunos términos son encontrados en partes de instrumentos de uso diario como cuchillo, hacha, canoa y remo. Algunas de estas similitudes son las siguientes.

11. a. Casa

pãi-tsewe

casa-diente

pãi tu'lũ-ti

casa oreja-CL.semilla

pãi talalaka-pi

casa costilla-CL.lineal

pãi i-wana

casa 3-brazo

pãi-ţa'na-ti

casa-boca-CL.cilíndrico'

pãi-tfanati-'tsa-ti

casa-boca.ABS-LOC-CL.cilíndrico

pãi-jãku

b. Navaja

Ǩthĩ-'tãi

cuchillo-pequeño

Ǩtht̃-'tãi

cuchillo-pequeño

k̃tht̃-'tãi

cuchillo-pequeño

K̃thĩ-'tãi

cuchillo-pequeño

3-boca

i-tana-ka

tiu-'tari

i-pĩjũ-naku 'pãi'

'viga (Lit. diente de la casa)'

'arete de la casa'

'paredes (Lit. costillas de la casa)'

'su brazo (brochal) de la casa'

'puerta (Lit. boca de la casa)'

'umbral de una puerta'

'sala (Lit. interior de la casa)'

'Lit. cuchillo-pequeño'

'punta (Lit. boca de la navaja)'

3-ala-CL.largo 'Iomo (Lit. espalda de la navaja)'

cabeza-CL.redondo 'mango'

3-pescuezo-CL.LOC.interno 'talón, empuñadora' 


\begin{tabular}{|c|c|c|}
\hline c. Hacha & & ja'wai \\
\hline $\begin{array}{l}\text { ja'wai } \\
\text { hacha }\end{array}$ & $\begin{array}{l}\text { i-numa } \\
\text { 3-boca }\end{array}$ & 'filo del hacha' \\
\hline $\begin{array}{l}\text { ja'wai } \\
\text { hacha }\end{array}$ & $\begin{array}{l}\text { i-'kati } \\
\text { 3-pierna }\end{array}$ & 'mango (Lit. pierna del hacha)' \\
\hline $\begin{array}{l}\text { ja'wai } \\
\text { hacha }\end{array}$ & $\begin{array}{l}\text { i-tulu-'naku } \\
\text { 3-oreja-CL.LOC.interno }\end{array}$ & 'su oído del hacha'4 \\
\hline d. Canoa & 'itsa & \\
\hline $\begin{array}{l}\text { 'itsa } \\
\text { canoa }\end{array}$ & $\begin{array}{l}\text { j-'kiri } \\
\text { 3-naríz }\end{array}$ & 'su proa (Lit. nariz de la canoa)' \\
\hline $\begin{array}{l}\text { 'itsa } \\
\text { canoa }\end{array}$ & $\begin{array}{l}\text { ¡-tana-ka } \\
\text { 3-ala-CL. largo }\end{array}$ & 'su casco (Lit. dorso/espalda)' \\
\hline $\begin{array}{l}\text { 'itsa } \\
\text { canoa }\end{array}$ & $\begin{array}{l}\text { i-ki'sa-pi } \\
\text { 3-labio-CL.lineal }\end{array}$ & 'sus bordas (Lit. labios de la canoa)' \\
\hline $\begin{array}{l}\text { 'itsa } \\
\text { canoa }\end{array}$ & $\begin{array}{l}\text { i-naku-'ti } \\
\text { 3-CL.LOC.interno-CL.cilí }\end{array}$ & 'su fondo de la canoa' \\
\hline
\end{tabular}

\section{Conclusiones}

En este artículo se presentó una breve descripción relacionada con cómo se estructuran morfológicamente los términos que aluden a las partes del cuerpo humano en la lengua y la cultura mehinaku. Se verificó, considerando los datos hasta ahora disponibles, que los términos de las partes del cuerpo son subcategorizados por el rasgo [+posesión]. O sea, la gramática de la lengua mehinaku exige que este tipo de nombres deben ocurrir, en la comunicación, con la mención de un determinado poseedor. En contraste, si no hubiera mención del poseedor, entonces estos términos serán marcados por el sufijo $\{-i\}$ 'ABSOLUTO', o 'no poseído'

También, con base en la morfología de la lengua, los términos de partes del cuerpo humano en mehinaku son factibles de ser sub-agrupados en simples y complejos. Los primeros constituidos simplemente por una base nominal seguida por el sufijo $\{-i\}$ 'ABSOLUTO', o bien, precedida, por un clítico pronominal que indica al poseedor. Los términos complejos, al contrario, se pueden formar por medio de bases nominales, seguidas, o no, por morfemas clasificadores, y precedidas por los clíticos pronominales de persona poseedora.

Otra característica relevante de algunos términos de partes del cuerpo humano en mehinaku es su extensión para nombrar partes de aves, mamíferos, peces, e inclusive para la denominación de determinadas partes de

\footnotetext{
${ }^{4}$ En la cultura occidental es conocido como 'ojo del hacha'
} 
la casa tradicional xinguana, partes de una canoa, hacha, canivete, entre otros.

Un aspecto que todavía precisa de una mejor comprensión se relaciona con el comportamiento semántico-gramatical de los morfemas: (i) -taku 'área', superficie', (ii) -naku 'interior, dentro', (iii) -penu 'sobre, encima. Estos morfemas que participan de la formación de palabras complejas, morfosintácticamente se asemejan a los clasificadores y tentativamente fueron glosados, en este texto, como clasificadores locativos. Al respecto, Aikhenvald (2000), Aikhenvald y Green (1998) mencionan que clasificadores locativos son muy raros, pero _ como explican las autoras _ algunos casos son registrados en América del Sur, como en las lenguas palikur y lokono (arawak del Norte), en lenguas karib y en dâw (nadahup). En todo caso, los tres morfemas citados no indican 'partes de', pero se refieren a un área o a una posición (Enfield 2006).

\section{Abreviaturas}

1, 2, 3: primera, segunda y tercera persona, singular y plural, respectivamente; ABS: Absoluto; ATRB: Atributivo; CL: Clasificador; CL.LOC: Clasificador locativo; CL.PRONM: Clítico pronominal.

\section{Referencias}

Aikhenvald, Alexandra Y. 1994. Classe nominal e gênero nas línguas aruák, Boletim do Museu Paraense Emílio Goeldi 10: 137-259.

Aikhenvald, Alexandra Y. 2000. Classifiers. A typology of noun categorization devices, Oxford, Oxford University Press.

Aikhenvald, Alexandra Y. y Diana Green. 1998. Palikur and the typology of classifiers. Anthropological Linguistics, 40: 429-480.

Andersen, Eliane S. 1978. Lexical universals of body-part terminology, en Joseph Greenberg (ed.) Universals of human language, Vol. 3, Word Structure, Stanford, Stanford University Press: 315-368.

Corbera Mori, Angel. 2011. Aspectos da morfofonologia e morfologia nominal da língua mehinaku (arawak), en Bruna Franchetto (org.). Alto Xingu: Uma sociedade multilíngue, Rio de Janeiro: Museu Nacional-FUNAI: 193215. Disponible en: http://www.etnolinguistica.org/xingu, p193-216

Enfield, N. J. 2006. Elicitation guide on parts of the body, Language Sciences 28: 148-157. doi: 10.1016/lj.langsci.2005.11.003

ISA. 2017 Mehinaku. [en línea]. Disponible en: https://pib.socioambiental.org/pt/povo/mehinako Consultado 23/01/2017.

McClure, Erica F. 1975. Ethno-anatomy: The structure of the domain, Anthropological Linguistics, 17: 78-88.

Meira, Sérgio. 2006. Tiriyó body part terms, Language Sciences, 28: 262-279. doi: $10.1016 /$ j.langsci.2005.11.009

Payne, David. 1991. A classification of maipuran (arawakan) languages based on shared lexical retentions, en D. C. Derbyshire y G. K. Pullum (eds.) Handbook of Amazonian languages, Vol. 3, Berlin: Mouton de Gruyter: 355-363. 